\title{
Effect of hemodialysis on the hearing function of patients with chronic renal failure
}

\author{
Akeem O. Lasisi $^{1}{ }^{*}$; Babatunde L. Salako ${ }^{2}$; Oladunni Osowole ${ }^{3}$; Wemimo P Osisanya ${ }^{1}$; Mohammed A Amusat ${ }^{2}$ \\ 1. Department of Otorhinolaryngology, 2. Department of Medicine- Nephrology Unit; 3. Department of Health \\ Promotion. - College of Medicine, University of Ibadan, Ibadan, Nigeria \\ * Corresponding author: P.O. Box 22040, University of Ibadan, Ibadan, Nigeria; Email: sakeemng@yahoo.com
}

\section{SUMMARY}

\begin{abstract}
Sensorineural hearing impairment (SHI) has been reported in chronic renal failure (CRF) patients with a prevalence of $20-40 \%$. The aetiopathogenetic mechanisms reported included osmotic alteration resulting in loss of hair cells and in some, complications of haemodialysis have been hypothesized We have in the past reported 2 cases of CRF patients who developed acute SHL following hemodialysis. This is a report of investigation of the effect of hemodialysis on the hearing function of CRF patients using pure tone audiometry findings. Thirty-three CRF patients were recruited for Pure Tone Audiometry (PTA) at admission and after three sessions of hemodialysis. The pure tone audiometry was done with a computer audiometer BA 20 Kamplex in the sound - proof (acoustic) booth in the ENT clinic. The duration of illness, dosage of diuretics and blood pressure were also noted. Similar age and sex-matched control were selected among volunteers who were otherwise clinically healthy. The data was processed using the Statistical Package for the Social Sciences (SPSS Inc, Chicago, Illinois, USA). Thirty-three CRF patients treated with haemodialysis and 28 healthy controls completed the study, 34 males and 27 females, age range was 16 - 72 years, mean of 45.30 (SD16.20) for subjects and 49.7 for controls. Hearing loss was found in 22/34 $(67 \%)$ at recruitment and $27 / 34(79 \%)$ after 3 sessions of hemodialysis. There was a significant difference between the mean pre- and posthemodialysis PTA values, $\mathbf{P}=\mathbf{0 . 0 0 0 8}$. There was also a significant correlation between post hemodialysis hearing threshold and (i) duration of illness $(P=0.00340)$ and (ii) creatinine levels of the patients $(P=\mathbf{0 . 0 3 5})$. In conclusion, there was a significant depression in the hearing threshold of patients with CRF following three sessions of hemodialysis. This could be caused by changes induced by hemodialysis or effects of the duration and severity of disease.
\end{abstract}

[Afr J Health Sci. 2006; 13:29-32]

\section{Introduction}

Sensorineural hearing impairment (SHI) has been reported in chronic renal failure $(\mathrm{CRF})$ patients with a prevalence of $20-40 \%$ [1]. The aetiopathogenetic mechanisms reported included osmotic alteration resulting in loss of hair cells, collapse of the endolymphatic space, oedema and atrophy of specialized auditory cells and in some, complications of haemodialysis have been hypothesized [2-4]. The role of hemodialysis in the causation of sensorineural hearing loss (SHL) is controversial; some authors have reported a depression in hearing threshold after hemodialysis while others are of the opinion that there was no relation between the two [4-6]. We have in the past reported 2 cases of CRF patients who developed acute SHL following hemodialysis [7]. This is a report of investigation of the effect of hemodialysis on the hearing function of CRF patients using pure tone audiometry findings and compared with controls.

\section{Methods}

Thirty-three CRF patients were recruited for Pure Tone Audiometry (PTA) at admission and after three sessions of hemodialysis. The pure tone audiometry was done with a computer audiometer BA 20 Kamplex in the sound - proof (acoustic) booth in the ENT clinic. The hearing acuity was measured in $\mathrm{db}$ at the frequencies $500-8000 \mathrm{~Hz}$. The average for the four frequencies $500 \mathrm{~Hz}, 1000 \mathrm{~Hz}, 2000 \mathrm{~Hz}$ and $4000 \mathrm{~Hz}$ were recorded. The duration of illness, dosage of diuretic and blood pressure were also 
noted. A similar number of age and sex matched control were selected among volunteers who were otherwise clinically healthy and had pure tone audiometry done and recorded. The data was processed using the Statistical Package for the Social Sciences (SPSS Inc, Chicago, Illinois, USA). The mean hearing acuity of pre- and post - hemodialysis were determined and paired student $\mathrm{t}$ - test was used to find the significance of the difference. Pearson correlation coefficient was used to find the correlation between PTA and other variables such as duration of illness, blood pressure, serum creatinine, dosage of diuretics and age. The inclusion criteria was consecutive CRF patients who had not had hemodialysis prior to inception of the study. The exclusion criteria included CRF patients who have been previously treated with hemodialysis or have uraemic encephalopathy or severe illness precluding patients from responding at audiometry and those with history of chronic otitis media.

\section{Results}

Thirty-three CRF patients treated with hemodialysis and 28 healthy controls were included in the study. This included, 34 males and 27 females, age range was 16-72 years, mean of $45.30(\mathrm{SD}=16.20)$. The duration of illness was 3-9 months, mean 6.4. The diastolic blood pressure was between 90 and 250 $\mathrm{mmHg}$, mean $113.64(\mathrm{SD}=21.33)$. The patients were on daily dose of diuretics (furosemide) between 40mg and 200mg; mean $102.42(\mathrm{SD}=66.29)$.

Hearing loss was found in $22 / 34(67 \%)$ at recruitment and 27/34 (79\%) after hemodialysis. The pre - hemodialysis hearing threshold was between 15 and $60 \mathrm{db}$, mean $37.42(\mathrm{SD}=18.55)$ and the posthemodialysis hearing threshold was between 25 and 90, mean 48.48( $\mathrm{SD}=20.33)$. Among the controls hearing threshold was between $10 \mathrm{db}$ and $70 \mathrm{db}$ with a mean of $35 \mathrm{db}$. There was a significant difference between the mean pre- and post- hemodialysis, $(\mathrm{t}=$ $5.115, \mathrm{P}=0.0008)$. There was a significant correlation between post-hemodialysis hearing threshold and (i) duration of illness $(\mathrm{P}=0.00340)$ and (ii) creatinine level of the patients $(\mathrm{P}=0.035)$. There was no significant correlation between post- hemodialysis hearing threshold with age $(\mathrm{P}=0.479)$, the diastolic blood pressure $(\mathrm{P}=0.056)$ and dosage of diuretics $(\mathrm{P}=-0.155)$.

\section{PTA pre- and post- haemodialysis against control}

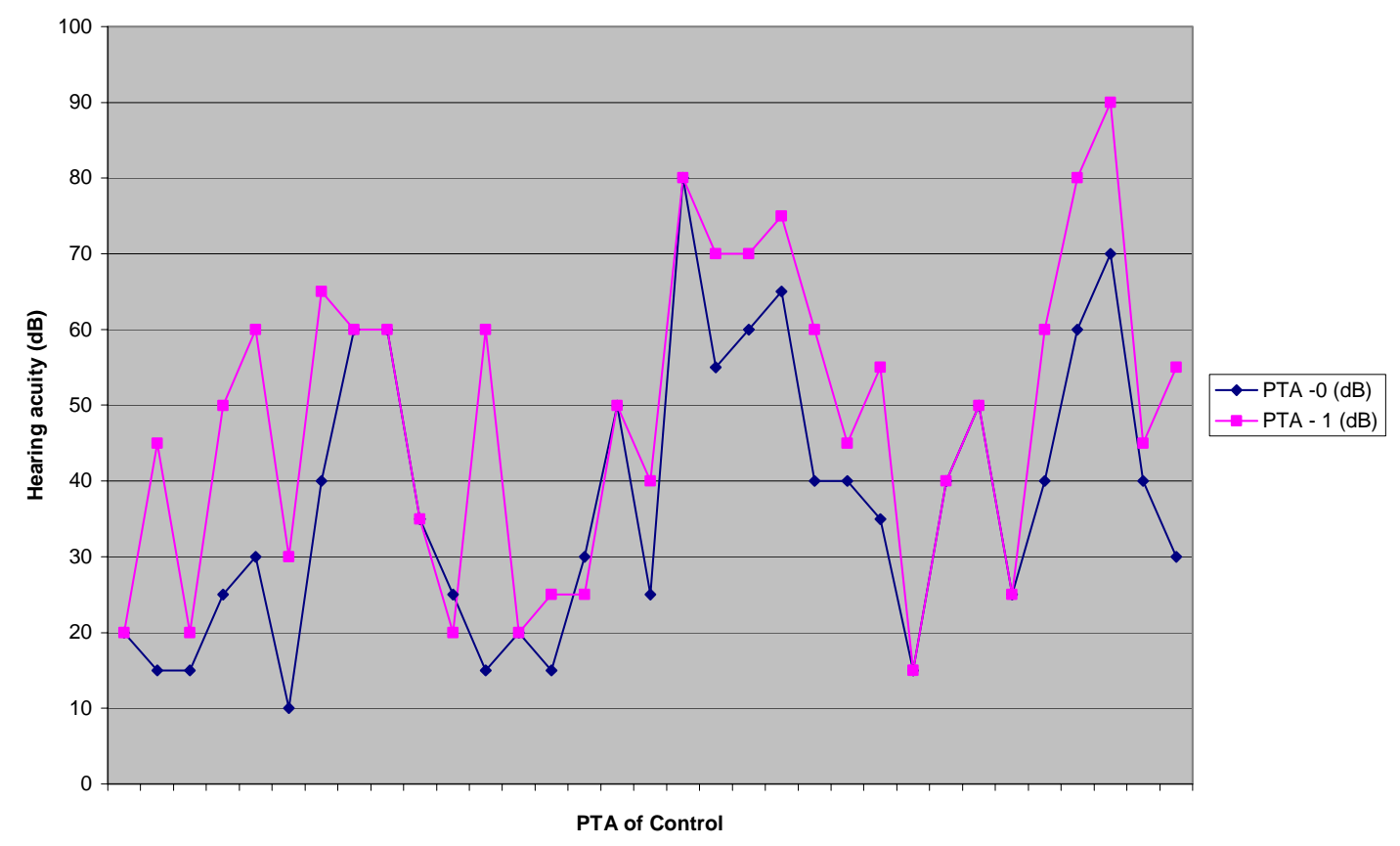

\section{Discussion}

The cochlea in patients under the treatment with hemodialysis has been reported to be susceptible to various insults and the patients frequently exhibit some degree of sensorineural hearing loss, although the responsible etiological factor(s) still remain controversial [5,6]. In addition, the concurrent existence of these factors in each patient make conclusions difficult on the significance of each 
variable. The high proportion of hearing loss at recruitment also confirms depressive effect of CRF on the hearing function. However, our study has shown significant depression in hearing threshold after 3 sessions of hemodialysis. Also, the presence of correlation between the post-treatment pure tone threshold and duration of illness and serum creatinine levels which was not found with the dosage of diuretics and blood pressure is similar to the report of Gatland et al [5] and Hutter et al [6]. Gatland et al [5] reported decrease in threshold in 22/31 ears after dialysis but found no correlation with weight changes. The fluctuation was said to be due to treatment induced changes in fluid and electrolyte composition of endolymph. Hutter et al [6] reported acute onset hearing loss and neurologic symptoms within 24 hours after haemodialysis in 7 patients. They concluded that the injury was associated with exposure to aged cellulose acetate membranes of dialyzers, allowing acetate degradation product to enter the blood. Temporal bone study revealing severe loss of hair cell in the organ of corti after polybrene administration have further confirmed the ototoxic effect of polybrene and other macromolecular substances [8,9]. We suspect that the observed depression in hearing threshold after hemodialysis in this study could be due to a combination of changes in fluid and electrolyte composition of endolymph and possibly exposure to aged cellulose acetate membranes of dialyzers used, allowing acetate degradation product to enter the blood, however this may need further confirmation with temporal bone study. Nikolopoulos et al [10] reported sensorineural hearing loss in 14/46 patients $(30.4 \%)$ after hemodialysis. Hearing loss was more in the hemodialysis group than in the peritoneal dialysis group, although, there were no significant changes in audiometric findings before and after haemodialysis.

In contrast SHL have not been found to be associated with hemodialysis by other workers. Stravoulaki et al [4] reported that no significant changes in PTA thresholds or Distortion product otoacoustic emission (DPOAE) amplitudes were encountered in renal patients before and after a HD session $(\mathrm{P}>0.05)$ in young patients undergoing haemodialysis, although SHL of unknown aetiology was found in $55.5 \%$ of cases. Similarly, Ozturan et al [11] has concluded that there was no relationship between SHL and hemodialysis following a study of 15 subjects and 10 controls using PTA and DPOAE before and after hemodialysis, however, high frequency hearing loss was found but there was no difference between controls and subjects. In another related study, Serbetcioglu et a [12] also concluded that single session of hemodialysis had no significant adverse effect on hearing in a study of 19 patients with chronic renal failure undergoing hemodialysis. This conclusion was also supported by the study of Kusakari et a [13]. Most previous studies did not find correlation between audiometric threshold and other variables such as blood pressure, use of ototoxic drugs, weight changes, haematocrit and metabolic bone disease. This is similar to our findings, although our result may have been influenced by the use of audiometer as the only audiologic modality used to assess the patients while some other investigators used DPOAEs and PTA [4, 8-11]. They reported that DPOAE seem to be more sensitive to incipient cochlear damage than behavior thresholds [4, 8-11]. We had no facility for DPOAE in our center.

\section{Conclusion}

From this report it was concluded that there was a depression in the hearing threshold of patients with CRF following three sessions of hemodialysis. This could be caused by changes induced by hemodialysis and the duration and severity of disease. However, there was no correlation with blood pressure and dosage of diuretics.

\section{References}

1. Quick CA, Fish A and Brown C. The relationship between the cochlea and the kidney. Laryngoscope. 1973, 83:1469-1482.

2. Johnson DW, Mathog RH. Hearing function in chronic renal failure. Annals of OtoRhinoLaryngology. 1976; 85:43-49.

3. Davison AM, Cameron JS, Grünfeld JP, Kerr DNS, Ritz E, Winearls CG. Oxford Textbook of Clinical Nephrology, 2nd edn. Oxford University Press, Oxford, UK, 1998.

4. Stavroulaki P, Nikolopoulos TP, Psarommatis I, Apostolopoulos N. Hearing evaluation with distortion-product otoacoustic emissions in young patients undergoing haemodialysis. Clinical Otolaryngology and Allied Sciences. 2001; 26:235-242.

5. Gatland D, Tucker B, Chalstrey C, Keene M and Baker L. Hearing loss in chronic renal failurehearing threshold changes following haemodialysis. Journal of the Royal Society of Medicine. 1991; 84: 87-589.

6. Hutter JC, Kuehnert MJ, Wallis RR, Lucas AD, Sen S \& Jarvis WR. Acute onset decreased vision and hearing traced to hemodialysis treatment with aged dialysers. Journal of the American Medical Association. 2000; 283:21282134.

7. Lasisi OA, Salako BL, Kadiri S, Arije A, OkoJaja R, Ipadeola A and Olatoke F. Sudden 
sensorineural hearing loss and hemodialysis. Journal of Ear, Nose and Throat. In press.

8. Kimura ET, Young PR, Barlow GH. A study of low and high molecular weights of hexadimethrine bromide - an antiheparin agent. Proceedings of Social and Experimental Biology. 1962; 111: 37-42.

9. Ransome J, Ballantyne JC, Shaldon S, Bosher SK and Hallpike CS. Perceptive deafness in subjects with renal failure treated with haemodialysis and polybrene. A clinicopathologic study. Journal of Laryngology and Otology. 1966; 80:651-677.

10. Nikolopoulos TP, Kandiloros DC, Segas JV, Nomicos PN, Ferekidis EA, Michelis KE, Apostolopoulos NJ, Adamopoulos GK.Auditory function in young patients with chronic renal failure. Clinical Otolaryngology and Allied Sciences. 1997; 22:222-225.

11. Ozturan $\mathrm{O}$ and Lam $\mathrm{S}$. The effect of haemodialysis on hearing using pure - tone audiometry and distortion - product otoacoustic emissions. Oto-RhinoLaryngology Journal of Otorhinolaryngology and Related Specialities. 1998; 60: 306-313.

12. Serbetcioglu MB, Erdogan S, Sifil A.Effects of a single session of hemodialysis on hearing abilities: Acta Otolaryngology. 2001; 121:836838.

13. Kusakari J, Hara A, Takeyama M, Suzuki S \& Igari $\mathrm{T}$. The hearing of the patients treated with haemodialysis: a long term follow-up study. Auris Nasus Larynx. 1992; 19:105-113. 